\title{
Mécanismes de la régulation du trafic membranaire
}

Le fonctionnement de toutes les cellules nécessite une intense activité de transport intracellulaire et de fusion de membranes dont les mécanismes commencent tout juste à être compris. Plusieurs protéines (ou familles de protéines) impliquées dans ces phénomènes ont déjà été identifiées, notamment les éléments du manteau entourant certaines vésicules, les protéines régulatrices liant le GTP et des molécules qui semblent intervenir lors de la fusion entre membranes. De plus, des réactions de phosphorylation-déphosphorylation jouent également un important rôle de régulation - notamment au cours de la mitose, où l'activation de la kinase p34 ${ }^{\text {cdc2 }}$ semble être associée à une inhibition du transport membranaire.

\section{Jean Gruenberg}

\section{ADRESSE ET TIRÉS À PART}

J. Gruenberg: chef de groupe. Laboratoire curopéen de biologie moléculaire (EMBL), Meyerhof strasse 1, Postfach 10.2209, D-6900 Heidelberg, Allemagne.

$\mathrm{m} / \mathrm{s} n^{\circ} 2$ vol. 9 , féurier 93 es différents compartiments subcellulaires sont des lieux de passage obligatoire aussi bien sur la voie de la biosynthèse (ou de la sécrétion) que sur celle de l'endocytose (ou de l'internalisation) (figure 1) ([1-3] et article de S. Méresse et al., p. 148 de ce numéro). Dans le premier cas, les protéines sont synthétisées dans le réticulum endoplasmique, puis transportées vers l'appareil de Golgi, où ont lieu toute une série de modifications post-traductionnelles. Finalement, les protéines atteignent le réseau transgolgien (trans-Golgi network), où elles sont triées et orientées vers leur destination ultérieure, qui va être la membrane plasmique, les granules de sécrétion ou les endosomes. En revanche, dans la voie de l'endocytose, les molécules internalisées apparaissent d'abord dans les endosomes précoces situés au voisinage de la membrane plasmique. A partir de là, ces molécules sont soit rapidement recyclées vers la surface cellulaire, comme c'est le cas pour plusicurs récepteurs, soit transportécs vers les endosomes tardifs, localisés dans la région périnucléaire près du Golgi. Certaines molćcules, en particulier les récepteurs des hydrolases lysosomiales, sont alors recyclées vers le réseau trans-golgien, alors que les molécules qui vont être dégradées sont empaquetées dans les lysosomes.

Depuis le travail de Palade et de ses collaborateurs sur l'étude de la voic de la biosynthèse [4], il est généralcment admis que le transport entre différents compartiments s'effectue par l'intermédiaire de vésicules membranaires (vesicular traffic). La membrane source s'invagine progressivement de manière à former un puits (pit) qui, finalement, se détache (budding), donnant naissance à une vésicule libre. Cette vésicule est entourée d'une membrane constituée des protéines et lipides destinés à être transportés, et elle contient les composés solubles qui étaient présents dans la lumière du compartiment donneur. La vésicule est ensuite adressée (targetting), avec un haut degré de spéci- 


\section{RÉFÉRENCES}

1. Griffiths G, Gruenberg J. The arguments for pre-existing early and late endosomes. Trends Cell Biol 1991; 1 : 5-9.

2. Murphy RF. Maturation models for endosome and lysosome biogenesis. Trends Cell Biol $1991 ; 1$ : 77-82.

3. Mellman I, Simons K. The Golgi complex : in vitro veritas? Cell $1992 ; 68$ : 829-40.

4. Palade G. Intracellular aspects of the process of protein secretion. Science $1975 ; 189$ : 473-85.

5. Griffiths G, Back R, Marsh M. A quantitative analysis of the endocytic pathway in baby hamster kidney cells. J Cell Biol 1989 ; 109 : 2703-20.

6. Davoust J, Cosson P. The dynamics of clathrin coats in living cells measured by advanced fluorescence microscopy. NATO ASI Ser $1992 ; 62$ : 27-42.

7. Hicke L, Schekman R. Molecular machinery required for protein transport from the endoplasmic reticulum to the Golgi complex. BioEssays $1990 ; 12$ : 253-8.

8. Goda Y, Pfeffer SR. Cell-free systems to study vesicular transport along the secretory and biosynthetic pathways. FASEB ./ 1989 ; 3 : 2488-94.

9. Balch WE. Biochemistry of interorganelle transport. J Biol Chem $1989 ; 264: 16965-8$.

10. Gruenberg J, Howell KE. Membrane traffic in endocytosis : insights from cell-free assays. Annu Rev Cell Biol 1989 ; 5 : 453-81.

11. Goldstein JL, Brown MS, Anderson RGW, Russel DW, Schneider WJ. Receptor-mediated endocytosis. Annu Rev Cell Biol 1985; 1 : 1-40.

12. Pearse B, Robinson MS. Clathrin, adaptors and sorting. Ann Rev Cell Biol $1990 ; 6: 151-72$

13. Morris SA, Ahle S, Ungewickell E. Clathrin-coated vesicles. Curr Op Cell Biol $1989 ; 1$ : 684-90.

14. Waters MG, Griff IC, Rothman JE. Proteins involved in vesicular transport and membrane fusion. Curr Op Cell Biol 1991; 3 : 615-620.

15. Pfeffer SR, Rothman JE. Biosynthetic protein transport and sorting by the endoplasmic reticulum and Golgi. Annu Rev Biochem 1987 ; 56 : 829-52.

16. Donaldson JG, Lippincott-Schwartz J, Klausner R. Guanine nucleotides modulate the effects of brefeldin $\mathrm{A}$ in semipermeable cells : regulation of the association of a

$110-\mathrm{kl}$ peripheral membrane protein with the Golgi apparatus. J Cell Biol $1991 ; 112$ : ficité, vers la membrane du compartiment receveur, avec laquelle elle fusionne. A l'état stationnaire, le nombre de vésicules assurant le transport entre deux compartiments est relativement faible, reflétant la rapidité du processus. On a pu, par exemple, démontrer, d'une part, que la membrane plasmique produit environ 1000 vésicules de $0,1 \mu \mathrm{m}$ de diamètre par minute, en déterminant le volume internalisé par endocytose [5], et, d'autre part, que la demi-vie d'une vésicule est inférieure à une minute [6]. Des vitesses de cet ordre impliquent que les composés requis doivent alors être recyclés avec une grande efficacité.

Des études génétiques chez la levure [7] et la reconstitution in vitro de différentes étapes du transport intracellulaire [8-10] ont permis d'identifier différentes catégories de protéines constituantes de la machinerie du trafic membranaire. Ces protéines peuvent être regroupées comme suit : les protéines impliquées dans le mécanisme de la fusion, comme le facteur

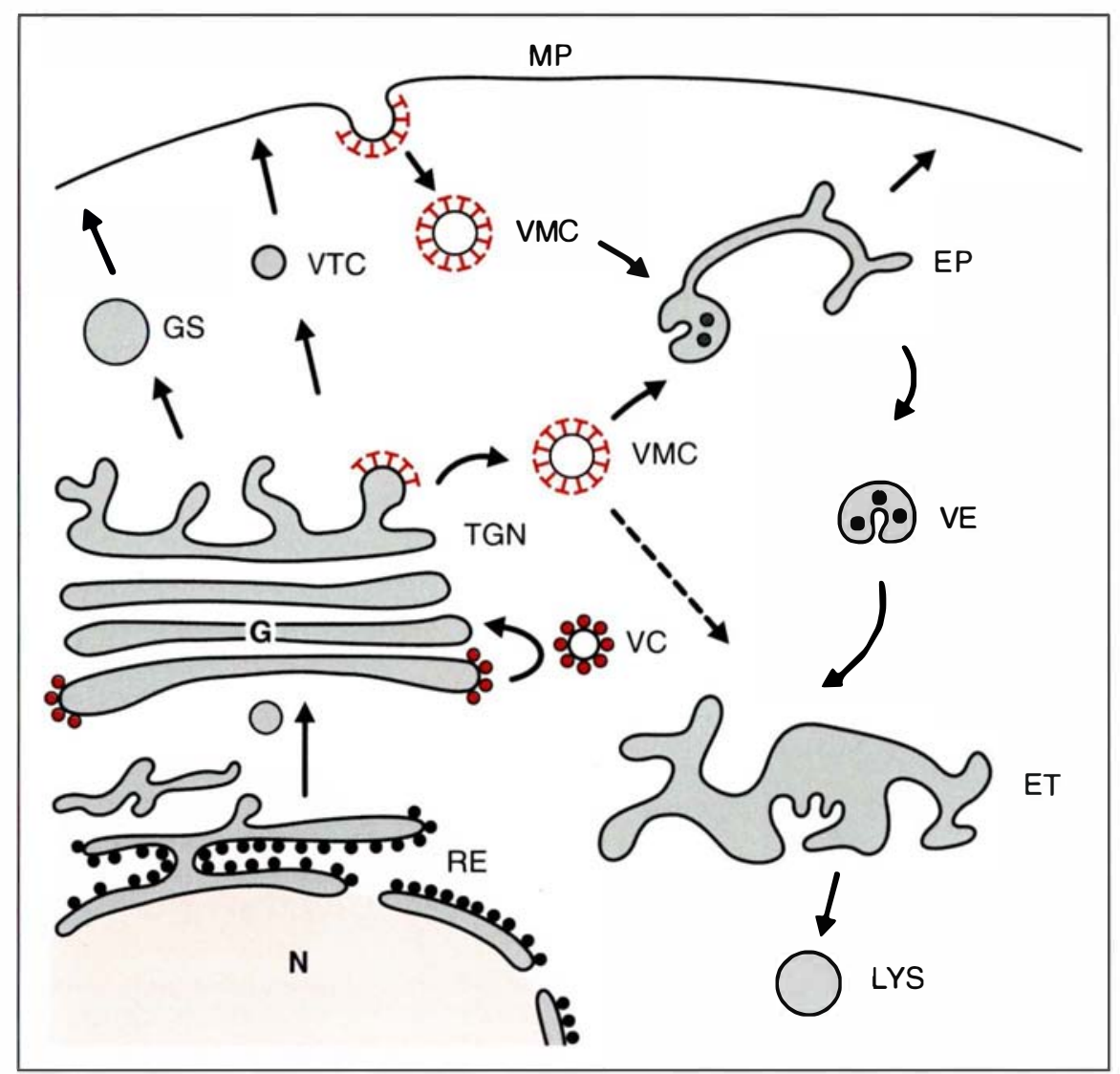

Figure 1. Voies de trafic membranaire. Les principales voies de transport membranaire dans une cellule animale sont indiquées. Dans la voie de la biosynthèse, les protéines synthétisées dans le réticulum endoplasmique (RE) sont transportées vers le Golgi (G), dans la région du noyau (N). Des vésicules (VC) entourées d'un manteau protéique différent de la clathrine et constitué de coatomers sont impliquées dans le transport intragolgien. Du Golgi, ces protéines sont ensuite transportées vers le réseau transgolgien (TGN), où elles sont triées puis orientées vers leurs destinations ultérieures, les granules de sécrétion (GS) dans certaines cellules, la membrane plasmique (MP) via des vésicules de transport constitutif (VTC), ou les endosomes via des vésicules mantelées par la clathrine (VMC). Lors de l'endocytose, les protéines internalisées sont transportées par des VMC vers les endosomes précoces (EP), d'où certains récepteurs sont rapidement recyclés vers la membrane plasmique (MP). Les molécules destinées à être dégradées apparaissent alors successivement dans des vésicules endosomiales (VE) à l'aspect typiquement multivésiculaire, puis dans les endosomes tardifs (ET), enfin dans les lysosomes (LYS). 
NSF (N-ethylmaleimide sensitive fusion protein) et les protéines qui lui sont associées, certains éléments régulateurs du transport qui font partie de la superfamille des protéines liant le GTP (GTP-binding proteins) et, enfin, certaines protéines du manteau cytoplasmique des vésicules de transport (coat proteins). Cette liste n'est pas exhaustive. En effet, nous n'avons pas mentionné certains candidats protéiques qui ne sont pas encore caractérisés. De plus, il est très probable que plusieurs éléments de la machinerie du transport membranaire n'ont pas encore été identifiés. Toutefois, l'ensemble des travaux faits à ce jour montre déjà que, dans les différentes étapes de transport, l'architecture de cette machinerie protéique est hautement conservée.

\section{Protéines du manteau}

\section{Clathrine et adaptateurs}

Grâce à des études de microscopie électronique, un manteau de 5-10 nm d'épaisseur a été identifié à la face cytoplasmique de certaines vésicules de transport. Ce manteau est constitué de protéines spécifiques, dont les micux étudiées à cc jour sont la clathrine et le complexe de protéines, appelé "adaptateur", qui lui est associé $([11,12]$ et article de S. Méresse et al., p. 148 de ce numéro). La clathrine recouvre au moins deux populations de vésicules différentes, impliquées respectivement, dans l'endocytose des récepteurs de surface et de leurs ligands (receptor-mediated endocytosis) et dans le transport des hydrolases lysosomiales entre le réseau transgolgien et les endosomes (figure 1). Un complexe adaptateur différent est associé au manteau de chaque type de vésicule. Toutefois, les constituants polypeptidiques de ces deux complexes sont très semblables, non seulement par leurs masses moléculaires et leurs stoechiométries, mais aussi par leurs séquences [12, 13].

Pourquoi ces vésicules s'entourentelles d'un manteau de clathrine? Toute une série de travaux récents suggèrent que les adaptateurs sont impliqués dans le tri (sorting) des protéines membranaires destinées à être transportées [12, 13]. Ils interagiraient spécifiquement avec les domaines cytoplasmiques de ces protéines, de manière à les rassembler (clustering) dans le puits en formation, à l'exclusion des protéines dites résidentes. Une seconde fonction du manteau de clathrine serait de fournir, lors de sa polymérisation, la force motrice nécessaire à l'invagination de la membrane. On ne sait pas si cette force est suffisante pour ensuite détacher la vésicule de la membrane, ou si une activité catalytique est alors requise. Après formation de la vésicule, le manteau se dépolymérise rapidement, laissant la vésicule dénudée, et les composants du manteau, libérés dans le cytosol, sont recyclés vers la membrane de départ afin d'être réutilisés. Il n'est pas exclu, toutefois, que certains polypeptides du manteau puissent rester associés aux vésicules, pour éventuellement jouer un rôle lors de l'adressage ou de la fusion.

\section{Coatomers}

Des travaux récents ont montré que les vésicules impliquées dans le transport intragolgien possèdent aussi un manteau protéique. Celui-ci ne contient pas de clathrine mais est constitué de complexes protéiques appelés coatomers, dont les sous-unités ont été nommées protéines cop (pour coat proteins) [14]. Le rôle fonctionnel de ces vésicules dans le transport intragolgien semble être différent de celui des vésicules mantelées par la clathrine. Certaines expériences indiquent que le transport dans le Golgi est non sélectif, impliquant que les passagers protéiques nc sont pas triés mais passivement inclus (bulk flow) [15], contrairement aux protéines transportées par les vésicules recouvertes de clathrine. Bien que la fonction des coatomers soit relativement mal connue, ces observations laissent présager que les manteaux des vésicules de transport peuvent jouer, du moins en partie, des rôles différents. Au-delà de ces différences, les coatomers et les protéines du manteau de clathrine semblent avoir néanmoins des points communs au niveau moléculaire. L'étude d'inhibiteurs de la voie de sécrétion, la bréfeldine $\mathrm{A}$ et le GTP $\mathrm{S}$, montre que l'association des coatomers avec les membranes golgiennes peut être réversible, suggérant qu'ils sont recyclés entre membrane et cytosol, tout comme les composés du manteau de clathrine [16-18]. De plus, les masses moléculaires des polypeptides du coatomer rappellent cclles de la clathrine et de ses adaptateurs [14]. Finalement, la séquence de $\beta$-cop, un composé du coatomer, présente une certaine homologie de séquence avec la $\beta$-adaptine, un composé des adaptateurs [19-20]. Ces observations suggèrent que les manteaux des différentes vésicules de transport sont bâtis d'après une structure commune, même si leurs fonctions different.

\section{Protéines de la sous- famille Ypt 1p/Sec4p/rab}

La superfamille des protéines liant le GTP, déjà impliquée dans de nombreux processus cellulaires, joue aussi un rôle important dans le trafic membranaire [21]. Des expériences génétiques chez la levure ont, tout d'abord, révélé l'existence de deux gènes, YPT1 et SEC4, qui sont requis chacun dans une étape différente de la voie de la biosynthèse [22, 23]. Ces gènes codent pour des petites protéines $(20-30 \mathrm{kDa})$ liant le GTP et homologues du protooncogène Ras. Depuis lors, de nombreuses protéines homologues de SEC4/YPT1 ont été découvertes et classées en quatre sous-familles principales [24]: YPT1/SEC4/Rab et ARF/Sar, qui sont impliquées dans la régulation du transport membranaire [25-27], Ras et Rho.

Dans les cellules de mammifères, près de vingt protéines $\mathrm{Rab}$, homologues de Ypt $1 p$ et Sec4p, ont déjà été identifiées [28-30], et ce nombre va vraisemblablement croître. Ces protéines sont associées à la face cytoplasmique des membranes par addition d'une chaîne aliphatique de type géranylgéranyl sur une cystéine présente à l'extrémité C-terminale [31]. Quelques protéines $\mathrm{Rab}$ ont déjà été localisées par immunocytochimie, et chacune est localisée de manière spécifique à la surface d'un ou de deux compartiments seulement $[27,32]$. Un signal présent dans la région Cterminale hypervariable garantit la spécificité de cette association [33], suggérant l'existence de récepteurs membranaires. Dans le cas de plusieurs protéines $\mathrm{Rab}$, il a maintenant été démontré in vitro [34-36] et in vivo 


\section{RÉFÉRENCES}

17. Orci L, Tagaya M, Amherdt M, et al. Brefeldin A, a drug that blocks sccretion, prevents the assembly of non-clathrin-coated buds on Golgi cistcrnac. Cell 1991; 64 : 1183-95.

18. Robinson MS, Krcis TE. Recruitment of coat proteins into Golgi membrancs in intact and permeabilized cells : effects of brefeldin $\mathrm{A}$ and $\mathrm{G}$-protcin activators. Cell 1993 ; 69 : 129-38.

19. Duden R, Griffiths G, Frank R, Argos $\mathrm{P}$, Krcis TE. $\beta-\mathrm{COP}$, a protein associated with non-clathrin coated vesicles and the Golgi complex, shows homology to $\beta$ adaptin. Cell 1991; 64 : 649-65.

20. Scrafini T, Stenbeck G, Brecht A, et al. A coat subunit of Golgi-derived nonclathrin-coated vesicles with homology to the clathrin-coated vesicle coat protein $\beta$ adaptin. Nature 1991; 349 : 215-20

21. Bourne HR, Sanders DA, McCormick F. The GTPasc superfamily: a conserved switch for diverse ccll functions. Nature 1990 ; 348 : 125-32.

22. Gallwitz D, Donath C, Sander C. A ycast gene encoding a protcin homologous to the hum an C-has/bas proto-oncogene product. Nature 1983 ; 306 : 704-7.

23. Salminen A, Novick PJ. A ras-like protein is required for a post-Golgi event in ycast sccretion. Cell 1987 ; 49 : 527-38.

24. Valencia A, Chardin P, Wittinghof er A, Sander C. The ras protein family: cvolutionary trec and role of conserved amino acids. Biochemistry 1991; 30 : 4637-48.

25. Balch WE. From $G$ minor to $G$ major. Curr Biol 1992 ; 2 : 157-60.

26. Pfcffer S. GTP-binding proteins in intracellular transport. Trends Cell Biol 1992 ; 2 : 41-6.

27. Gruenberg J, Claguc M. Regulation of intracellular membrane transport. Curr Op Cell Biol 1992 ; 4 : 593-9.

28. Touchot N, Chardin P, Tavitian A. Four additional members of the ras superfamily isolated by an oligonuclcotid stratcgy : molecular cloning of YPT-related cl)NAs from a rat brain library. Proc Natl Acad Sci USA 1987 ; 84: 8210-4.

29. Zahraoui A, Touchot N, Chardin P, Tavitian A. The hum an $\mathrm{R}$ ab gencs encode a family of GTP-binding proteins related to yeast YPT1 and SEC4 products involved in secretion. J Biol Chem 1989; 264 : 12384-401.

30. Chavrier $P$, Vingron $M$, Sander $C$, Simons K, Zerial M. Molecular cloning of YPT1/SEC4-rclated clDNAs from an epithelium cell line. Mol Cell Biol $1990 ; 10$ : 6578-85

31. Evans T, Hart MJ, Cerionc RA. The ras superfamilies: regulatory proteins and post-translational modifications. Curr Op Cell Biol 1991; 3 : 185-91.

32. Goud B, McCaffrey M. Small GTPbinding proteins and their role in transport. 144
[37] que chacune contrôle effectivement une étape du transport membranaire.

Quelle est la fonction des protéines de la sous-famille SEC4/YPT4/Rab? L'analogie avec Ras, dont la structure est connue [38-40], permet de prédire que ces protéines, ainsi que toutes les protéines homologues de Ras, présentent deux conformations différentes, selon qu'elles lient le GTP ou le GDP. Grâce à ce changement de conformation, ces protéines peuvent fonctionner comme une forme d'interrupteur moléculaire (molecular switch), et introduire une vectorialité dans un processus biologique. A la suite des travaux sur SEC4 chez la levure [41], Bourne avait proposé que les protéines de cette sous-famille contrôlent la spécificité de l'adressage à différentes étapes du transport [42]. Des études plus récentes sur Yptlp sont en accord avec ce modèle $[43,44]$. Afin de maintenir la vectorialité du processus d'adressage, l'échange GDP/GTP ainsi que l'hydrolyse du GTP lié doivent alors être contrôlés par des protéines spécifiques. Des candidates pour ce rôle ont, depuis, été identifiées pour Ras, pour des membres de la famille SEC4/YPT1/Rab, ainsi que pour d'autres petites protéines liant le GTP [31].

Toutefois, il n'est pas du tout évident que tous les membres de cette sousfamille soient fonctionnellement équivalents à SEC4 et YPT1. Dans la mesure où la fonction précise des protéines Rab est mal connue, il est possible d'envisager que la classification actuelle a amalgamé dans la même sous-famille des groupes de protéines aux fonctions distinctes. De plus, plusieurs isoformes de certaines protéines $\mathrm{Rab}$, codées par des gènes différents, ont été découvertes dans les mêmes cellules (par exemple Rab1a et b, Rab3a et $b$, et Rab4a et $b$ ). On ne sait pas encore si chaque isoforme a une fonction spécifique ou bien si les différentes isoformes de la même protéine sont redondantes (voir ci-dessous dans le cas de Rab1 et Rab4).

\section{Protéines de la sous- famille ARF/sar}

En plus des protéines de type Ypt1p/Sec4p/Rab, les protéines ARF sont aussi impliquées dans le transport membranaire. A l'origine, ARF (ADP-ribosylation factor) a été décrite comme étant un cofacteur nécessaire à la ribosylation de la sous-unité activatrice stimulatoire $(\mathrm{G} \alpha \mathrm{s})$ de l'adénylate cyclase par la toxine du choléra [45-47]. Depuis lors, plusieurs protéines ARF ont été identifiées chez la levure et dans les cellules de mammifères [48]. Il est maintenant évident que ces protéines sont, en réalité, des composantes de la machinerie du transport membranaire, et qu'elles sont impliquées à plusieurs étapes [48-53]. Toutefois, leur fonction précise dans le transport membranaire n'est pas clairement déterminée. Les travaux de Serafini et al. [50] suggèrent qu'une protéine $A R F$ contrôlerait l'assemblage et le démantèlement du manteau de coatomers sur les vésicules de transport dans le Golgi, par l'intermédiaire de protéines contrôlant l'échange GDP/GTP et l'hydrolyse du GTP lié.

Enfin, des travaux chez la levure montrent que la protéine Sarlp, qui présente un certain degré d'homologic avec ARF, est nécessaire au transport entre le réticulum endoplasmique et le Golgi [54-56]. Cette étape de transport a pu être disséquée génétiquement chez la levure [7], une approche difficilement réalisable chez les eucaryotes supérieurs. Ainsi, deux groupes de gènes, intitulés mutants de classe I ou II, ont été identifiés. Les premiers contrôlent la "formation " de vésicules de transport (bourgeonnement et fission), et les seconds la "consommation" de ces vésicules (adressage et fusion). Le gène SAR 1 fait partie des mutants de type I et est lié génétiquement à SEC12, un autre gène de la même classe. De plus, la protéine codée par SAR1 (Sar1p), qui n'est pas modifiée posttraductionnellement, interagit avec une protéine membranaire, le produit du gène SEC12, lors de la formation de vésicules $[54,56]$.

\section{Fonctions des petites protéines liant le GTP}

Comme nous venons de le voir, l'ensemble de ces travaux suggère que différentes sous-familles de petites protéines liant le GTP sont impli- 
quées dans le transport membranaire. Les observations faites à ce jour suggèrent que ces protéines contrôlent la formation de vésicules (Sar1p), l'assemblage-démantèlement du manteau (ARF), l'adressage (Yptlp/ Sec4p), et, éventuellement, la fusion elle-même. Il est donc tentant de spéculer que, à chaque étape de transport, la succession d'interactions entre protéines cytosoliques (par exemple, le manteau) et protéines membranaires (par exemple, Sec12p) est contrôlée séquentiellement par les protéines des sous-familles YPT1/SEC4/Rab et ARF/Sar, qui agiraient comme des éléments modulaires de la machinerie de transport.

\section{Protéines $\mathbf{G}$ hétéro- trimériques}

Outre ces petites protéines liant le GTP, une série d'observations très récentes indiquent que les grandes protéines $G$ hétérotrimériques sont aussi impliquées dans le transport membranaire [25-27]. La fonction de ces protéines dans la transduction des signaux extracellulaires par des récepteurs de surface a été largement étudiée [21]. Une première indication suggérant que des protéines $G$ pourraient aussi intervenir dans le contrôle du transport intracellulaire est venue de l'observation que le fluorure d'aluminium inhibe toute une série d'étapes de transport. En effet, ce produit qui active les protéines $G$ hétérotrimériques semble sans effet sur les petites protéines liant le GTP [57]. Plus directement, Stow et al. [58] ont montré que la surexpression de $\mathrm{G} \alpha_{\mathrm{i} 3}$ retarde la sécrétion constitutive de protéoglycanes. De plus, cet effet est annulé en présence de la toxine de Pertussis qui catalyse une ADP-ribosylation spécifique des sous-unités $\mathrm{G} \alpha_{\mathrm{i}}$. Finalement, la formation in vitro de vésicules de sécrétion à partir du réseau transgolgien, qui est sensible au fluorure d'aluminium, est stimulée par la sous-unité G $\beta \gamma$ purifiée et par ribosylation de $\mathrm{G} \alpha_{\mathrm{i} 3}$ en présence de toxine de Pertussis [59]. Par ailleurs, la fusion des endosomes entre eux semble aussi dépendre de protéines G [60].

A l'heure actuelle, il est difficile de proposer un modèle pour le rôle des $m / s n^{\circ} 2$ vol. 9, féurier 93 protéines $G$ hétérodimériques dans la régulation du transport. Toutefois, le fait que ces processus soient sensibles à certaines toxines suggère que, les protéines $G$ impliquées interagissent avec des facteurs d'échange GDP/GTP. Lors de la transduction du signal, c'est la fixation du ligand sur des récepteurs membranaires appartenant à la superfamille de récepteurs à sept segments transmembranaires qui provoque l'échange du GDP par du GTP [61]. Bien qu'aucun récepteur de ce type n'ait été à ce jour impliqué dans la régulation du transport membranaire, le récepteur $\beta$ adrénergique, un archétype des récepteurs liés aux protéines $G$, peut être internalisé [62], et une autre protéine du même type est vraisemblablement recyclée entre le réticulum endoplasmique et le Golgi $[63,64]$. Il sera donc intéressant de déterminer si ces protéines membranaires peuvent être impliquées dans le contrôle du transport par l'intermédiaire de protéines G. De plus, l'effet des toxines stimulant l'ADPribosylation sur le transport permet une corrélation entre ARF et les protéines $G$, qui nous offre la première indication d'un couplage possible entre différents types de protéines liant le GTP.

\section{Régulation du trafic membranaire en mitose}

Dans les cellules de mammiferes, le transport membranaire lié aux voies de la biosynthèse et de l'endocytose est arrêté pendant la mitose [65]. Des expériences effectuées en système acellulaire indiquent que cet arrêt est contrôlé par des événements de phosphorylation-déphosphorylation. En effet, la fusion membranaire peut être inhibée par un cytosol mitotique. Cette inhibition est contrôlée par la protéine kinase $\mathrm{p} 34^{\text {cdc2 }}$ associée à la cycline $B$ [66, 67], qui règle l'entrée en mitose in vivo. L'invagination de vésicules mantelées par la clathrine est aussi inhibée par un cytosol mitotique [68].

Les cibles de la protéine kinase p34 cdc2 n'ont pas encore été identifiées. Toutefois, des travaux récents montrent que Rab4a et $\mathrm{Rab} 1 \mathrm{a}$ sont tous deux phosphorylés durant la mitose in vivo et par la protéine kinase $\mathrm{p} 34^{\mathrm{cdc} 2}$ in vitro [67]. Paradoxalement, les autres protéines de cette sous-famille ne le sont pas, suggérant que Rab4a et $\mathrm{R} a b 1 \mathrm{a}$ sont spécifiquement impliquées dans la régulation du trafic membranaire pendant la mitose. Il est intéressant de remarquer que ces deux protéines possèdent des isoformes ( $\mathrm{R} a b 1 \mathrm{~b}$ et Rab4b) qui ne sont pas, non plus, phosphorylées, suggérant, dans ce cas précis, l'existence d'une différence fonctionnelle entre les différentes isoformes de la même protéine Rab. L'utilisation d'inhibiteurs de phosphatase, en particulier l'acide okadaïque, suggère que des phosphorylations-déphosphorylations sont aussi impliquées dans la régulation du transport pendant l'interphase $[70,71]$. On ne sait cependant pas si certaines protéines $\mathrm{Rab}$ peuvent alors être phosphorylćes, ni si ces phosphorylations au cours de l'interphase et de la mitose utilisent des cibles ou des mécanismes communs.

\section{Mécanismes de la fusion entre membranes}

Les mécanismes de la fusion à proprement parler restent relativement peu connus. Les études faites avec des bicouches lipidiques artificielles montrent que leur contact est énergétiquement très défavorable à des distances inférieures à $2 \mathrm{~nm}$ [72]. A ces distances, une force de répulsion (hydration repulsion) se développe, qui est vraisemblablement due à l'organisation des molécules d'eau à la surface de la bicouche. Pour permettre la fusion, cette force doit être surpassée ou contournée. Cela pourrait s'effectuer en rendant la bicouche plus hydrophobe, par exemple grâce à l'insertion de molécules amphipatiques, comme l'ont suggéré des expériences effectuées avec des bicouches immobilisées sur des plaques de mica [73].

Dans le cas des fusions entre membranes biologiques, le rôle de cette force de répulsion n'est pas clairement démontré. Les modèles les plus couramment répandus suggèrent que deux membranes peuvent être pontées par l'oligomérisation de protéines membranaires, formant un "col" ou 
33. Chavrier P, Gorvel JP, Stelzer E, Simons K, Gruenberg J, Zerial M. Hypervariable $\mathrm{C}$-terminal domain of $\mathrm{r}$ ab proteins acts as a targetting signal. Nature 1991; $353:$ 769-72.

34. Gorvel JP, Chavrier P, Zerial $M$, Gruenberg J. Rab 5 controls early endosome fusion in vitro. Cell 1991; $64: 915-25$.

35. Plutner $\mathrm{H}$, Cox AD, Pind $\mathrm{S}$, et al. Rab1b regulates vesicular transport between the endoplasmic reticulum and successive Golgi compartments. J Cell Biol 1991 ; 115 : 31-43.

36. Fischer von Mollard G, Sudhof TC, Jahn R, A small GTP-binding protein dissociates from synaptic vesicles during exocytosis. Nature 1991 ; 349 : 79-81

37. Bucci C, Parton RG, Mather IH, et al. The small GTP-ase rab5 functions as a regulatory factor in the early endocytic pathway. Cell $1992 ; 70$ : 715-28.

38. De Vos A, Tong L, Milburn M, et al. Three-dimensional structure of an oncogene protein : catalytic domain of human C-Hras p21. Structure of ras proteins. Science 1988 ; 239 : 888-93.

39. Pai EF, Kabsch W, Krengel U, Holmes KC, John J, Wittinghofer A. Structure of the guanine-nucleotide-binding domain of the Ha-ras oncogene product $\mathrm{p} 21$ in the trisphosphate conformation. Nature 1989 ; 341 : 209-14.

40. Tong L, Milburn MV, de Vos AM, Kim SH. Structure of ras protein Science $1989 ; 245: 244$.

41. Goud B, Salminen A, Walworth NC, Novick PJ. A GTP-binding protein required for secretion rapidly associates with secretory vesicles and the plasma membrane in yeast. Cell $1988 ; 53: 733-68$.

42. Bourne H. Do GTPases direct membrane traffic in secretion? Cell $1988 ; 53$ : 669-71.

43. Rexach MF, Schekman RW. Distinct biochemical requirements for the budding, targeting and fusion of ER-derived transport vesicles. J Cell Biol 1991; 114 : 219-29.

44. Segev N. Mediation of the attachment or fusion step in vesicular transport by the GTP-binding Ypt1 protein. Science 1991; 252: 1553-6

45. Kahn RA, Gilman AG. The protein factor necessary for ADP-ribosylation of Gs by cholera toxin is itself a GTP-binding protein. J Biol Chem $1986 ; 263$ : 8282-7.

46. Kahn RA, Goddard C, Newkirk M. Chemical and immunological characterization of the $21 \mathrm{kDa}$ ADP-ribosylation factor of adenylate cyclase. J Biol Chem 1988 ; $263: 8282-7$

47. Schleifer LS, Kahn RA, Hanski E, Northup JK, Sternweis PC, Gilman AG. Requirements for cholera toxin-dependent ADP-ribosylation of the purified regulatory component of adenylate cyclase. J Biol Chem
48. Kahn RA, Kern FG, Clark J, Gelmann EP, Rulka C. Human ADP-ribosylation factor: a functionally conserved family of GTP-binding proteins. J Biol Chem 1991; 266 : 2606-14,

49. Kahn RA, Randazzo P, Serafini $T$, $e t$ al. The amino terminus of ADP-ribosylation factor (ARF) is a critical determinant of ARF activities and is a potent and specific inhibitor of protein transport. $J$ Biol Chem $1992 ; 267: 13039-46$

50. Serafini T, Orci L, Amherdt M, Brunner $M, K$ ahn RA, Rothman JE. ADPribosylation factor is a subunit of the coat of Golgi-derived COP coated vesicles : a novel role for a GTP-binding protein. Cell $1991 ; 67: 239-53$

51. Balch WE, Kahn RA, Schwaninger R. ADP-ribosylation factor is required for vesicular trafficking between the endoplasmic reticulum and the cis-Golgi compartment. $J$ Biol Chem 1992 ; 267 : 13047-52.

52. Lenhard JM, Kahn RA, Stahl PD. Evidence for ADP-ribosylation factor (ARF) as a regulator of in vitro endosome-endosome fusion. J Biol Chem 1992; 267 : 13047-52.

53. Taylor TC, Kahn RA, Melançon P. Two distinct members of the ADP ribosylation factor family of GTP-binding proteins regulate cell-free intra-Golgi transport. Cell 1993 (sous presse).

54. D'Enfert C, Wuestehube LJ, Lila T, Sche kman R. Sec $12 p$-dependent membrane binding of the small GTP-binding protein Sar1p promotes formation of transport vesicles from the ER. J Cell Biol 1991; 114: 663-70.

55. Nakano A, Muramatsu M. A novel GTP-binding protein, Sar1p, is involved in transport from the endoplasmic reticulum to the Golgi apparatus. J Cell Biol 1989 ; 109 : 2677-91.

56. Oka T, Nishikawa SI, Nakano A. Reconstitution of GTP-binding Sar1 protein function in ER to Golgi transport. J Cell Biol $1991 ; 114: 671-9$.

57. Kahn RA. Fluoride is not an activator of the smaller (20-25 kDa) GTP-binding proteins. J Biol Chem 1991; 266 ; 15595-7.

58. Stow JL, de Almeida JB, Narula N, Holtzmann EJ, Ercolani L, Ausiello DA. A heterotrimeric $\mathrm{G}$ protein, $\mathrm{G}$ ai-3, on Golgi membranes regulates the secretion of a heparan sulfate proteoglycan in LLC-PK1 epithelial cells. J Cell Biol 1991; 114: 1113-24.

59. Barr F, Leyte A, Mollner S, Pfeuffer T, Tooze S, Huttner WB. Trimeric G-proteins of the trans-Golgi network are involved in the formation of constitutive secretory vesicles and immature granules. FEBS Lett $1991 ; 293: 1-5$.

60. Colombo MI, Mayorga LS, Casey PJ Stahl PD. Evidence of a role for heterotrimeric GTP-binding proteins in endosome fusion. Science $1992 ; 255: 1695-7$.
61. Dohlman HG, Thorner J, Caron MG, Lefkowitz RJ. Model systems for the study of seven-transmembrane-segment receptors. Annu Rev Biochem 1991; 60: 653-88.

62. Raposo G, Dunia I, Delavier-Klutchko C, Kaveri S, Strosberg D, Benedetti EL. Internalization of $\beta$-adrenergic receptor in A431 cells involves non-coated vesicles. Europ. J Cell Biol 1989 ; 50 : 340-52.

63. Pelham HRB. Control of protein exit from the endoplasmic reticulum. Annu Rev Cell Biol 1989; 5 : 1-24.

64. Pelham HRB, Roberts LM, Lord JM. Toxin entry : how reversible is the secretory pathway? Trends Cell Biol $1992 ; 2$ : 183-5.

65. Warren G. Mitosis and membranes. Nature 1989 ; 342 : 857-8.

66. Tuomikoski $\mathrm{T}$, Felix $\mathrm{M}$, Doree $\mathrm{M}$, Gruenberg J. Inhibition of endocytic vesicle fusion in vitro by the cell-cycle control protein kinase cdc2. Nature $1989 ; 342$ : 942-5.

67. Thomas $\mathrm{L}, \mathrm{Clarke} \mathrm{P}, \mathrm{Pagano} \mathrm{M}$, Gruenberg J. Inhibition of membrane fusion in vitro via cyclin B but not cyclin A. J Biol Chem 1992 ; 267 : 6183-7.

68. Pypaert M, Mundy DI, Souter E, Labbe JC, Warren G. Mitotic cytosol inhibits invagination of coated pits in broken mitotic cells. J Cell Biol 1991; 114 : 1159-66.

69. Bailly E, McCaffrey M, Touchot N, Zahroui A, Goud B, Bornens M. Phosphorylation of two small GTP-binding proteins of the rab family by $\mathrm{p} 34 \mathrm{cdc} 2$. Nature $1991 ; 350: 715-8$,

70. Woodman P, Mundy DI, Cohen $P$, Warren G. Cell-free fusion of endocytic vesicles is regulated by phosphorylation. $J$ Cell Biol 1992 ; 16 : 331-8.

71. Davidson HW, McGowan $\mathrm{CH}$, Balch $W E$. Evidence for the regulation of exocytic transport by protein phosphorylation. J Cell Biol 1992 ; 116 : 1343-55.

72. R and RP, Parsegian VA. Hydration forces between phospholipid bilayers. Biochem Biophys Acta 1989 ; 988 : 351-76.

73. Helm CA, Israelachvili JN, McGuiggan PM. Role of hydrophobic forces in bilayer adhesion and fusion. Biochemistry 1992; 31 : 1794-805.

74. Almers W. Exocytosis. Annu Rev Physiol $1990 ; 52: 607-24$.

75. Bentz J, Ellens H, Alford D. An architecture for the fusion site of influenza hemagglutinin. FEBS Lett $1990 ; 276$ : 1-5

76. Blobel CP, Wolfsberg TG, Turck CW Myles DG, Primakoff P, White J. A potential fusion peptide and an integrin lig and domain in a protein active in sperm-egg fusion. Nature $1992 ; 356$ : 248-52.

77. Rothman JE, Orci L. Molecular dissection of the secretory pathway. Nature 1992 355 : 409-15 
un "pore " [74, 75]. Ces modèles prédisent qu'au début du processus le flux de lipides pourrait commencer le long des surfaces hydrophobes de ces protéines. Les meilleurs exemples de protéines qui induisent la fusion membrane-membrane sont les protéines membranaires de l'enveloppe de certains virus, en particulier la protéine HA de l'influenza. Lors de l'infection virale, le virus est internalisé par endocytose. Le $\mathrm{pH}$ acide de la lumière de l'endosome induit la fusion de son enveloppe avec la membrane endosomiale. Lors de ce processus, HA change de conformation, exposant une hélice $\alpha$ amphipatique d'environ 20 résidus qui était préalablement enfouie dans la protéine. Une hélice analogue a été identifiée dans la séquence de plusieurs virus, ainsi que, tout réccmment, dans la séquence de $\mathrm{PH} 30$, une protéine impliquée dans la fusion spermatozoïde-ovocyte [76].

Les fusions intracellulaires sont contrôlées par des mécanismes qui diffèrent de ceux requis lors des fusions virales (topologiquement extracellulaires). De plus, les composés de la machinerie intracellulaire sont vraisemblablement recyclés. On pourrait donc s'attendre à ce qu'une " protéine de fusion " intraccllulaire, si elle se dépliait lors de la fusion comme une protéine virale, ait ensuite la capacité de se replier, peut-être par l'entremise d'un mécanisme requérant de l'énergie. Rothman et al. ont identifié une protéine soluble (NSF ou $N$-ethylmaleimide-sensitive fusion protein) qui contient deux sites de liaison de l'ATP, et qui est nécessaire au transport intragolgien [77]. Cette protéine, ainsi que son homologue chez la levure (Sec18p), est aussi impliquée dans d'autres étapes de transport membranaire. Rothman et al. ont proposé que le complexe formé entre cette molécule et des protéines SNAP (soluble NSF associated protein) forme un des éléments d'une machinerie universelle de fusion de membranes intracellulaires. Toutefois, la fonction précise de NSF dans le transport et la fusion n'cst pas établie.

\section{Conclusion}

Plusieurs types de protéines composant la machineric du transport membranaire ont été identifiées, en particulier des protéines du manteau, le NSF et les protéines associćes, et, enfin, les protéines qui lient le GTP. Bien que certains aspects du transport membranaire soient maintenant étudiés dans le détail, la fonction de plusieurs composés essentiels n'est pas encore clairc. De plus, il est possible, sur la base d'études fonctionnelles, de prédire l'existence d'au moins deux catégories de récepteurs membranaires qui n'ont pas encore été identifiés. Ceux-ci doivent être requis, d'une part pour le recrutement spécifique de certains facteurs cytosoliques et, d'autre part, pour maintenir la spécificité des interactions membrane-membrane. De plus, les protéines catalysant la fusion à proprement parler ne sont pas connues. Les recherches en cours laissent, toutefois, présager que la dissection moléculaire de la machinerie protéique du transport sera bientôt possible

\section{Summary}

Mechanisms of membrane regulation

Over the last few years, several aspects of the mechanisms regulating membrane transport between different sub-cellular organelles have been studied at the molecular level. These studies have revealed different categories of molecules, in particular components of the proteinacous coat of transport vesicles, rcgulatory elements belonging to the GTPbinding protein super-family, and, finally, molecules presumably implicated in the mechanism of membrane fusion. It is also clear that phosphorylation-dephosphorylation events are somehow involved. These studies have already demonstrated that some common design remains conserved in the mechanisms controlling different steps of transport. However, several important components remain to be identificd. Whereas progress has been made in understanding the regulation of transport, for instance via GTP-binding proteins, very little is known about proteins that either mediate close interactions between membranes or that catalyse the fusion step itself. The current challenge is to unravel these complex regulatory mechanisms, and, to dissect the membrane transport machinery. 